\title{
Collateral Uterine Arterial Supply in the Setting of Various Uterine Pathologies
}

\author{
Christine E. Boone ${ }^{1} \quad$ Yilun Koethe $^{2}$ Maureen P. Kohi ${ }^{2}$ \\ ${ }^{1}$ Department of Medicine, Santa Clara Valley Medical Center, \\ San Jose, California, United States \\ 2Division of Vascular and Interventional Radiology, Department \\ of Radiology and Biomedical Imaging, University of California, \\ San Francisco, California, United States
}

Evan D. Lehrman²

\begin{abstract}
Address for correspondence Evan D. Lehrman, MD, Division of Vascular and Interventional Radiology, Department of Radiology, University of California, San Francisco, 505 Parnassus Avenue, San Francisco, CA 94117, United States (e-mail: Evan.Lehrman@ucsf.edu).
\end{abstract}

J Clin Interv Radiol ISVIR 2018;2:191-196

\begin{abstract}
Keywords

- uterine artery embolization

- collateral blood supply

- postpartum hemorrhage

- uterine arteriovenous fistula

- uterine fibroids

In most patients, bilateral uterine arteries supply the uterus exclusively. However, collateral arterial supply to the uterus has been described, predominantly in the context of uterine artery embolization (UAE) for fibroid treatment. This may lead to incomplete embolization, which can impact clinical outcomes. Therefore, identification of potential collateral supply to the uterus is essential for achieving optimal clinical results. The authors report three cases of collateral uterine arterial supply from the ovarian and round ligament arteries in the setting of uterine arteriovenous fistula (UAVF), postpartum hemorrhage (PPH), and symptomatic fibroids. Aortography from the level of the renal arteries was performed for initial evaluation of uterine arterial supply in all patients and identified hypertrophied, tortuous ovarian artery collaterals. Selective angiography of the external iliac artery was required to identify round ligament collateral uterine supply. Three-dimensional cone beam computed tomography (CT) was also used to visualize vascular distribution of bilateral uterine arteries and an ovarian collateral feeding a UAVF. In regard to outcomes, identification of collateral uterine arterial supply allowed for single-session complete embolization in fibroid patients with collateralized round ligament or ovarian arteries and in the PPH patient in disseminated intravascular coagulation with predominantly ovarian artery supply to the uterus. Identification of collateral uterine arterial supply facilitated technical success in all patients. In the setting of various uterine pathologies, identification of collateral uterine arterial supply is essential to optimize clinical success of UAE.
\end{abstract}

\section{Introduction}

For women with a variety of uterine pathologies, including symptomatic fibroids, postpartum hemorrhage (PPH), adenomyosis, and uterine arteriovenous fistula (uAVF), uterine artery embolization (UAE) provides a minimally invasive and uterine-sparing treatment option. In most patients, the uterine arteries exclusively provide the uterine arterial supply. ${ }^{1}$ However, collateral arterial supply to the uterus has been described, predominantly in the setting of UAE for fibroid therapy. ${ }^{1,2}$ Approximately 5 to $10 \%$ of patients with uterine fibroids have collateral supply from the ovarian arteries. ${ }^{1,3-5}$
Collateral supply from other sources, including the round ligament ${ }^{6,7}$ and inferior mesenteric arteries, ${ }^{3,8}$ have also been reported. In addition, undetected collateral blood supply to the uterus has been described as a possible cause of UAE clinical failure, ${ }^{9,10}$ and embolization of these collaterals has resulted in clinical success following initial failure. ${ }^{4}$ Therefore, recognition of potential collateral arterial supply to the uterus may be essential for achieving optimal clinical success in treating a variety of uterine pathologies. Herein, we report three cases of collateral uterine arterial supply in the setting of various uterine pathologies. received

July 7, 2018

accepted after revision

August 6, 2018

published online

December 7, 2018
DOI https://doi.org/

$10.1055 / \mathrm{s}-0038-1676166$

ISSN 2457-0214.
(C)2018 by Indian Society of

Vascular and Interventional

Radiology
License terms

()(1) $\ominus \circledast$ 


\section{Case Presentations}

\section{Case 1}

A 33-year-old female, with a history of thalassemia anemia and two prior cesarean deliveries underwent dilation and evacuation (D\&E). Two hours following the procedure, she experienced heavy vaginal bleeding, and a bedside ultrasound (US) demonstrated the presence of intrauterine clot. Her hematocrit decreased from 31 to $27 \%$, but she remained hemodynamically stable and underwent subsequent dilation and curettage (D\&C). Her bleeding increased intraoperatively, and the total estimated blood loss was 2,250 mL for both operations. Despite two doses of methylergonovine maleate to augment uterine contractions, two units of packed red blood cell (PRBC) transfusion, and placement of an intrauterine balloon, her hematocrit was $22 \%$, and she experienced dizziness and continued to hemorrhage vaginally. Thus, an urgent UAE was performed.

Following right transfemoral arterial access, an aortogram did not demonstrate any active extravasation. Pelvic aortogram illustrated that the uterine arterial supply was predominately from the ovarian arteries with minor contribution from the uterine arteries ( - Fig. 1). Ovarian arteries were found to provide $\sim 75 \%$ of the arterial supply to the uterus. Using a 3-French microcatheter (Hi-Flo Renegade; Boston Scientific) coaxially through a 5-French Cobra-2 (C2) catheter (Terumo), the uterine arteries were embolized to stasis with gelatin sponge (Gelfoam; Upjohn). Next, an SOS catheter (AngioDynamics) was used to successively catheterize each of the ovarian arteries. Using a Hi-Flo Renegade
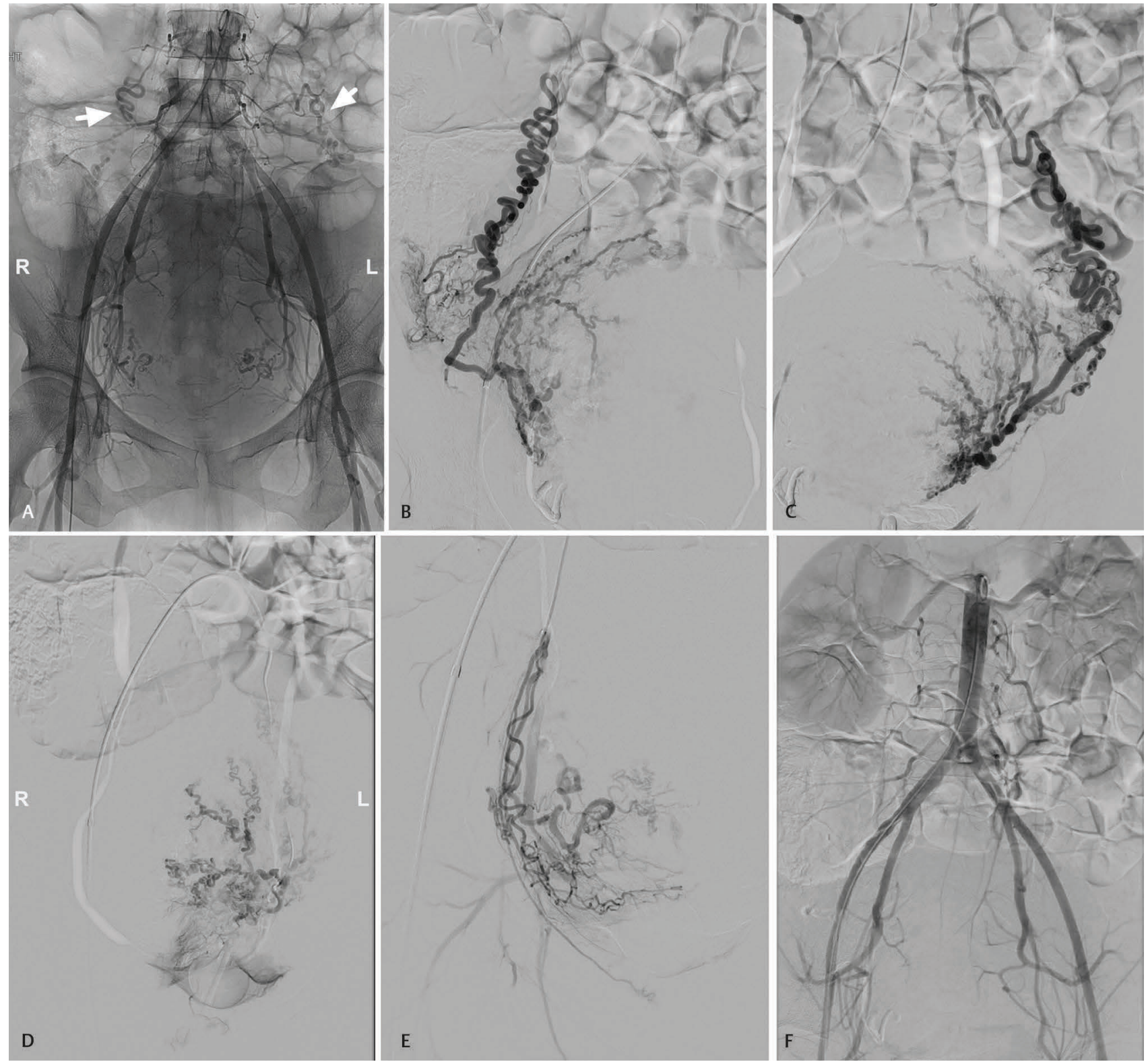

Fig. 1 Case 1: (A) Pre-embolization flush pelvic aortogram demonstrating bilateral uterine and ovarian (arrows) arterial supply to the uterus, (B) right ovarian arteriogram, (C) left ovarian arteriogram, (D) left uterine arteriogram, (E) right uterine arteriogram, and (F) post-embolization arteriogram with stasis of bilateral uterine arteries and bilateral ovarian arteries. L, left side; $R$, right side. 
microcatheter, Gelfoam embolization of the ovarian arteries to stasis was performed. Her postoperative course was complicated by lightheadedness and dizziness, for which she received additional transfusions of one unit of PRBC, two units of fresh frozen plasma, and one unit of cryoprecipitate. Her hematocrit 15 hours postoperatively was $23.5 \%$. She was discharged home in stable condition on the second post-procedure day, with a hematocrit of $33 \%$. At a clinical follow-up 16 days after the UAE, no additional episodes of vaginal bleeding had occurred.

\section{Case 2}

A portion of this case has been previously presented in a report of a transvaginal approach to embolize a large uAVF ${ }^{11}$ following two UAEs. Here, we present the details of those UAE procedures as an ovarian arterial collateral was found to feed the UAVF during them. This aspect of this case is has not previously been reported.

A 23-year-old female, with a history of D\&C and subsequent uAVF development, was transferred from an outside hospital for heavy vaginal bleeding. She desired future fertility, and the decision was made to treat her UAVF with UAE as opposed to hysterectomy. During the initial UAE, both uterine arteries were selectively embolized to angiographic stasis with 500 to $900 \mu \mathrm{m}$ tris-acryl gelatin microshperes (Embosphere; Merit Medical) and gelatin sponge. A right ovarian arteriogram showed collateral supply of the uterus without filling of the UAVF. Immediate post-procedure US did not demonstrate flow within the uAVF.

Two months later, the patient was hospitalized with recurrent vaginal bleeding and hypotension. The recurrence might have been due to embolization of the arteries feeding the $\mathrm{UAVF}$, instead of the arteriovenous communications of the uAVF. The uAVF would have been able to recruit new arterial feeders. Pelvic US showed recurrence of the uAVF. Pelvic aortogram with 5-French flush catheter again showed engorged uterine arteries and right ovarian artery ( - Fig. 2A). A 5-French SOS catheter was used to selectively catheterize the right ovarian artery, and a coaxial Hi-Flo Renegade microcatheter was used to perform arteriography. Early filling of the bilateral pelvic veins was present during subsequent right uterine artery injection ( - Fig. 2B). A three-dimensional (3-D) cone beam CT was performed to depict the right ovarian collateral supply ( - Fig. 2C). A 3-D cone beam CT was also utilized to study the right and left uterine arteries ( $\bullet$ Fig. 2B, C). Bilateral uterine arteries were embolized to stasis with Gelfoam sponge through coaxial Hi-Flo Renegade microcatheter and 5-French SOS catheter, since they were previously embolized with Embosphere and believed to induce less severe post-embolization syndrome compared with particles. The right ovarian artery was embolized to stasis with Gelfoam sponge as well. Final aortography did not demonstrate flow within the uAVF. Unfortunately, the patient did continue to have vaginal bleeding. After the third procedure, in which a percutaneous transvaginal approach was employed to embolize the uAVF directly, there was no further vaginal bleeding in the 17 months following the procedure. ${ }^{11}$

\section{Case 3}

A 41-year-old female presented to interventional radiology (IR) clinic with symptomatic uterine fibroids, causing dysmenorrhea, menorrhagia, urinary urgency, and pelvic pressure. Her magnetic resonance imaging (MRI) pelvis demonstrated numerous enhancing uterine fibroids. The enlarged uterus measured $11.7 \times 10.4 \times 10.1 \mathrm{~cm}$. Because she was not a myomectomy candidate and did not desire hysterectomy, a UAE was recommended.

Following right transfemoral arterial access, the left uterine artery was not well visualized on pelvic aortogram ( - Fig. 3A). The left internal iliac artery was selected using a C2 catheter, and left internal iliac arteriogram confirmed diminutive left uterine artery. Left external iliac arteriogram demonstrated sole left-sided uterine arterial supply from the left round ligament artery ( - Fig. $3 \mathrm{~B}$ ). The left round ligament was selectively catheterized with a 3-French microcatheter and was embolized to near stasis with 500 to 900 $\mu \mathrm{m}$ Embosphere ( - Fig. $3 \mathrm{C}$ ). The left uterine artery was not embolized as was not providing blood supply to the uterus. Next, the selectively catheterization and embolization of the right uterine artery was performed with Embosphere particles ranging in size from 500 to $900 \mu \mathrm{m}$. The patient tolerated the procedure well and was discharged the next day. Follow-up MRI at 5 months revealed a decrease in the size of the fibroids and lack of enhancement of all but one fibroid, which was located anterior, inferior, midline to slightly to the left relative to the endometrium. The uterus overall had decreased to $10.1 \times 8.9 \times 9.0 \mathrm{~cm}$.

\section{Conclusion}

Collateral arterial supply to the uterus may arise from many different sources. Ovarian arteries form anastomoses with the ascending branches of the uterine arteries in 10 to $15 \%$ of women. ${ }^{1,3-5}$ Other sources of collateral arterial supply to the uterus have been described including the round ligament artery, ${ }^{6,7}$ the inferior mesenteric artery (IMA), ${ }^{3,8}$ and an unnamed vessel arising from the infrarenal aorta. ${ }^{12}$ Two previous reports have described non-fibroid uterine pathology with collateral blood supply from other sources, including the round ligament artery in a patient with ${ }^{* *} \mathrm{PPH}^{7}$ and an ovarian artery arising from the IMA in a patient with adenomysosis. ${ }^{8}$

Perioperative imaging is an important factor in identifying collaterals in different uterine pathologies. Preoperative cross-sectional imaging, such as MR angiography (MRA), ${ }^{13}$ can facilitate procedure planning by allowing evaluation of vascular anatomy and potential collateral arterial supply to the uterus. While preoperative imaging should be utilized to improve procedural planning, preoperative imaging is not possible in all types of uterine pathology, especially in urgent situations, such as intractable uterine hemorrhage. In such cases, intraoperative imaging to examine the pelvic vascular anatomy is critical.

Intraoperative modalities are primarily angiography and three-dimensional cone beam CT; however, consensus regarding the optimal modality and timing of its use is yet to be reached. In this series, initial flush pelvic aortogram was 

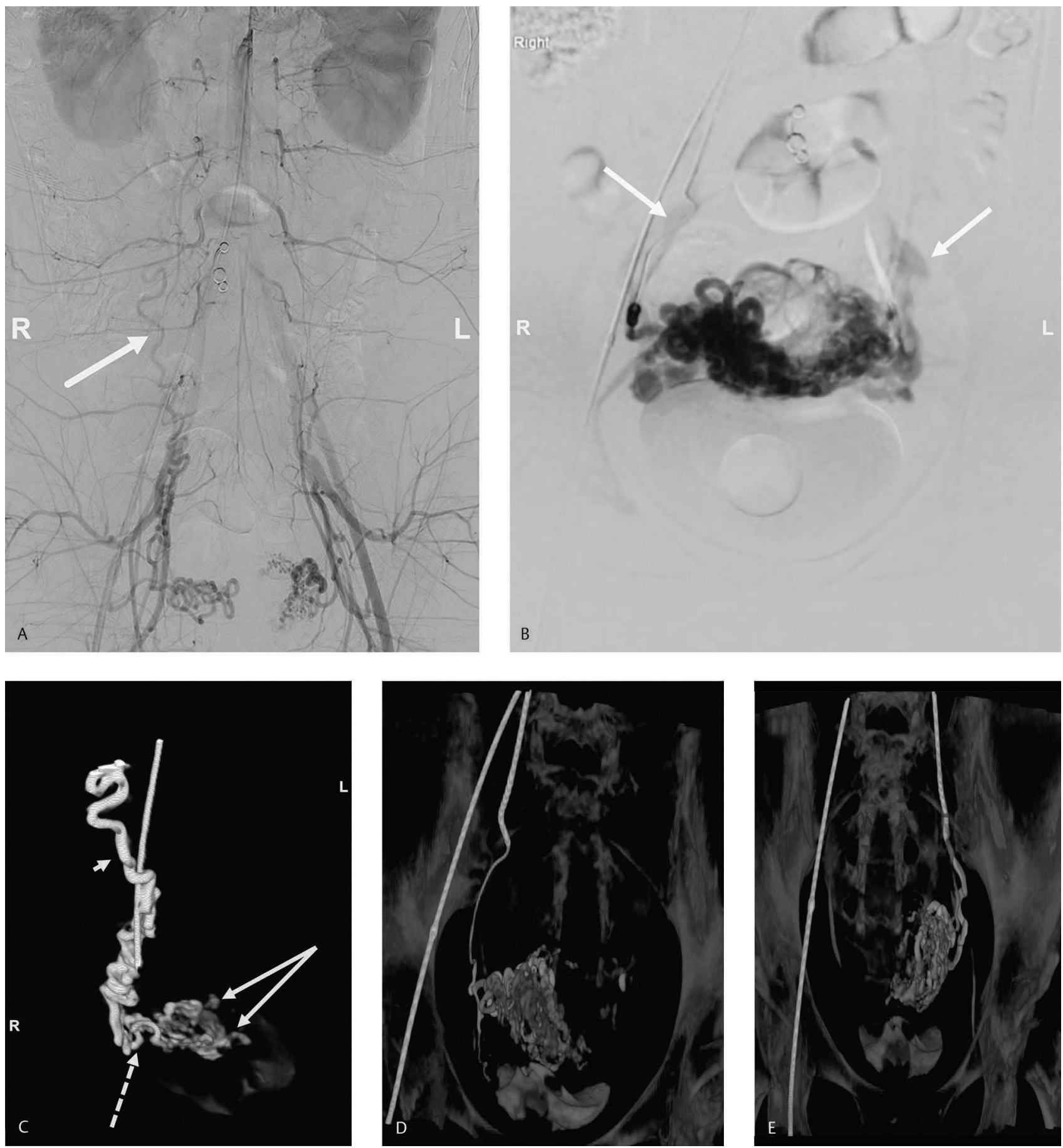

Fig. 2 Case 2: (A) Pre-embolization flush pelvic arteriogram demonstrating bilateral uterine and engorged right ovarian (arrow) arterial supply to the uterus. (B) 3-D cone beam CT reconstruction of right uterine arteriogram. (C) 3-D cone beam CT reconstruction of left uterine arteriogram. (D) 3-D cone beam CT reconstruction of right ovarian arteriogram showing enlarged, tortuous right ovarian artery (short arrow), with hypertrophied arterial inflow (dashed long arrow), and dilated venous channel representing the uAVF (solid long arrows). The image is slightly rotated right oblique anterior. (E) Early filling pelvic veins (arrows). 3-D, three-dimensional; CT, computed tomography; L, left side; $\mathrm{R}$, right side; uAVF, uterine arteriovenous fistula.

useful for examining pelvic vasculature and demonstrating anatomic variations; however, its routine use in UAE remains controversial. Three-dimensional cone beam CT was used in case 2 to study the unusual arterial distribution of the bilateral uterine arteries and ovarian collateral feeding the uterus and uAVF. Furthermore, as demonstrated in case 3, when the uterine artery is diminutive or absent, possible collateral arterial supply to the uterus should be investigated.

Imaging collaterals to the uterus is challenging, and different uterine pathologies may introduce distinct obstacles, such as more severely abnormal vascular anatomy in $\mathrm{UAVF}$ or having little or no preoperative information 


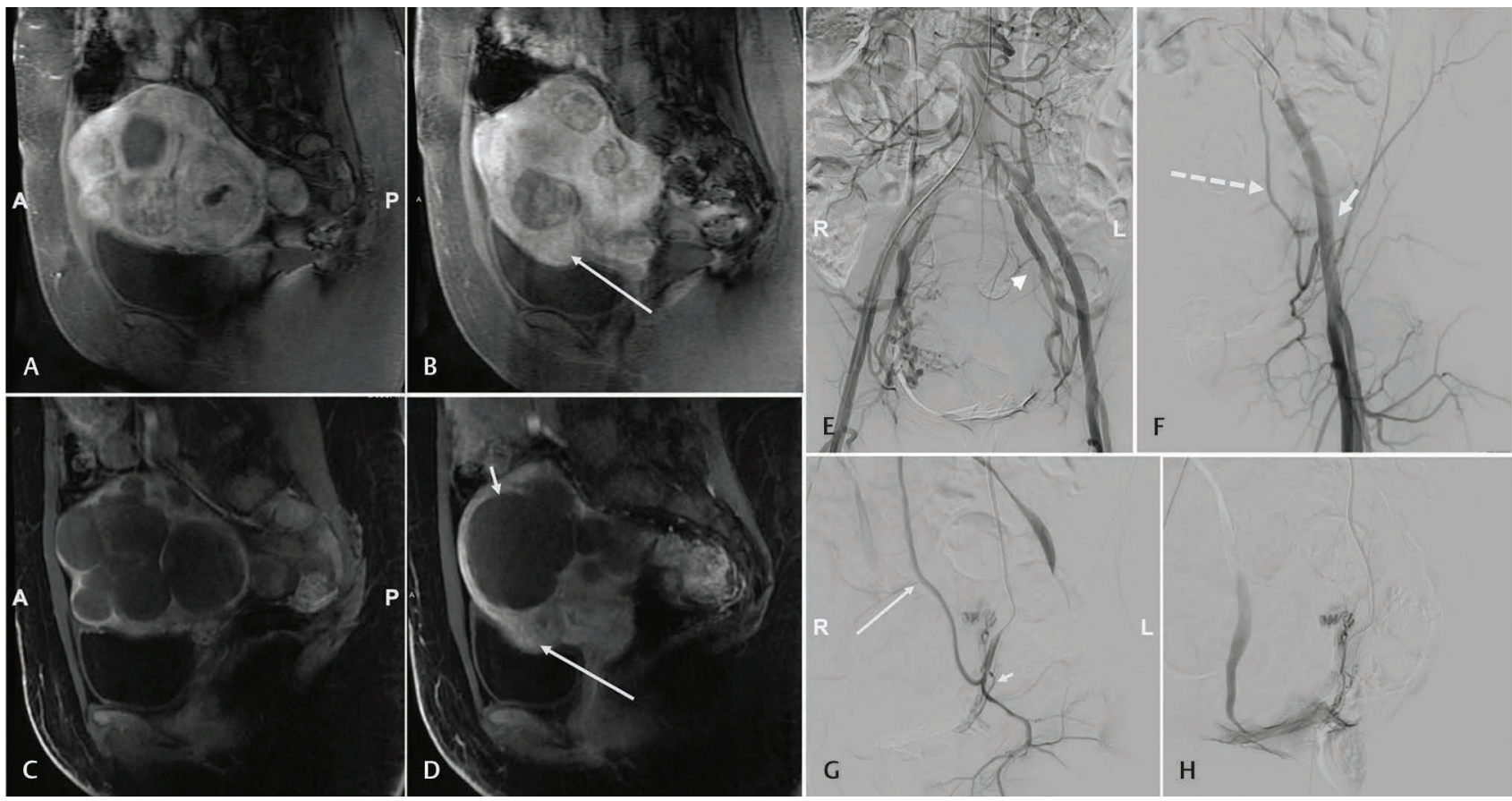

Fig. 3 Case 3: (A) Pre-embolization sagittal post-gadolinium MRI depicting enlarged uterus containing multiple heterogeneously enhancing fibroids. (B) Similar to A, with fibroid that remained enhanced post-UFE. (C) A 5-month post-embolization sagittal post-gadolinium MRI depicting non-enhancing fibroids. (D) Similar to $\mathbf{C}$, demonstrating the largest fibroid in the uterine fundus without any residual enhancement after treatment (short arrow) and the fibroid that remained enhanced after treatment (long arrow, same fibroid indicated with arrow in B). (E) Flush pelvic aortogram demonstrating right uterine artery supplying fibroids and minimal contribution from the left uterine artery. The left inferior epigastric artery extends superiorly (arrowhead). (F) Left external iliac arteriogram with inferior epigastric artery (dashed long arrow) and common femoral artery (short arrow). (G) Selective arteriogram of left external iliac artery branch demonstrates the inferior epigastric artery (long arrow) and origin of tortuous, enlarged left round ligament artery. (H) Post-selective catheterization of the left round ligament artery. A, anterior; L, left side; MRI, magnetic resonance imaging; P, posterior; R, right side; UFE, uterine fibroid embolization.

or planning time in an emergency, to finding potential collaterals. The authors found that both of these modalities allowed identification of different types uterine collaterals in diverse situations. Previous investigations of routine pelvic aortography in identification of uterine collaterals found sensitivity of only 18 to $25 \%^{4,14}$; these studies focused only on identifying ovarian artery collaterals in UAE for uterine fibroids. While flush pelvic aortogram may have low sensitivity for detecting ovarian arterial collaterals in treatment of uterine fibroids, that sensitivity may still provide significant clinical utility in a different situation, such as an emergent PPH. The efficacy of routine cone beam CT for detection of uterine collaterals has not yet been formally evaluated. An investigation into the utility of these modalities for identifying uterine collateral blood supply, in other uterine pathologies, or urgent situations, such as PPH, could facilitate the establishment of consensus or guidelines for optimal intraoperative imaging to improve the ability to rapidly assess pelvic vascular anatomy in emergent settings, where minimizing time to achieving stasis is vital.

In the setting of multiple uterine pathologies, such as fibroids, PPH, and uAVF, recognition and treatment of collateral uterine arterial supply are important to optimize clinical success of UAE. Differences in the pathophysiology or natural history of these conditions may present unique challenges in the identification of collateral blood supply to uterus and the impact of these collaterals on treatment outcome.

\section{Technical Notes}

- Institutional review board (IRB) exemption was obtained for this study on the basis of small number ( 3 or less) and de-identification of human subjects.

- All pelvic aortography used power injection of contrast at $10 \mathrm{~mL} / \mathrm{s}$ for a total volume of $20 \mathrm{~mL}$.

- All selective microcatheter digital subtraction angiography used power injection of contrast at $2 \mathrm{~mL} / \mathrm{s}$ for a total volume of $10 \mathrm{~mL}$.

\section{Availability of Data and Material}

Not applicable.

\section{Conflicts of Interest}

The authors declare that they have no conflict of interest.

\section{Funding}

None.

\section{Authors' Contributions}

Christine E. Boone performed the chart review and drafted the manuscript. Maureen P. Kohi conceived of the study and participated in its design and coordination. Maureen P. Kohi and Evan D. Lehrman identified cases for inclusion 
and edited manuscript. All authors read and approved the final manuscript.

\section{Acknowledgments}

None.

\section{References}

1 Pelage JP, Le Dref O, Soyer P, et al. Arterial anatomy of the female genital tract: variations and relevance to transcatheter embolization of the uterus. AJR Am J Roentgenol 1999;172(4):989-994

2 Pelage JP, Walker WJ, Le Dref O, Rymer R. Ovarian artery: angiographic appearance, embolization and relevance to uterine fibroid embolization. Cardiovasc Intervent Radiol 2003;26(3):227-233

3 Dixon S, Tapping CR, Chuah PS, Bratby M, Uberoi R, Anthony S. Successful fibroid embolization of pelvic and inferior mesenteric artery collaterals after previous uterine artery embolization. Acta Radiol 2012;53(3):292-295

4 White AM, BanovacF, Yousefi S, Slack RS, Spies JB.Uterine fibroid embolization: the utility of aortography in detecting ovarian artery collateral supply. Radiology 2007;244(1):291-298

5 Razavi MK, Wolanske KA, Hwang GL, Sze DY, Kee ST, Dake MD. Angiographic classification of ovarian artery-to-uterine artery anastomoses: initial observations in uterine fibroid embolization. Radiology 2002;224(3):707-712

6 Saraiya PV, Chang TC, Pelage J-P, Spies JB. Uterine artery replacement by the round ligament artery: an anatomic variant discovered during uterine artery embolization for leiomyomata. J Vasc Interv Radiol 2002;13(9 Pt 1):939-941
7 Wi JY, Kim H-C, Chung JW, Jun JK, Jae HJ, Park JH. Importance of angiographic visualization of round ligament arteries in women evaluated for intractable vaginal bleeding after uterine artery embolization. J Vasc Interv Radiol 2009;20(8):1031-1035

8 Smoger DL, Kancherla V, Shlansky-Goldberg RD. Uterine fundal blood supply from an aberrant left ovarian artery originating from the inferior mesenteric artery: implications for uterine artery embolization. J Vasc Interv Radiol 2010;21(6):941-944

9 Volkers NA, Hehenkamp WJK, Birnie E, et al. Uterine artery embolization in the treatment of symptomatic uterine fibroid tumors (EMMY trial): periprocedural results and complications. J Vasc Interv Radiol 2006;17(3):471-480

10 Nikolic B, Spies JB, Abbara S, Goodwin SC. Ovarian artery supply of uterine fibroids as a cause of treatment failure after uterine artery embolization: a case report. J Vasc Interv Radiol 1999;10(9):1167-1170

11 Lehrman ED, Heller M, Poder L, Kerlan R, Huddleston HG, Kohi MP. Transvaginal obliteration of a complex uterine arteriovenous fistula using ethylene vinyl alcohol copolymer. J Vasc Interv Radiol 2017;28(6):842-843

12 McLucas B. Collateral supply to uterine leiomyomata from an unnamed vessel: a case report. Minim Invasive Ther Allied Technol 2009;18(2):106-108

13 Worthington-Kirsch RL, Andrews RT, Siskin GP, et al. II. Uterine fibroid embolization: technical aspects. Tech Vasc Interv Radiol 2002;5(1):17-34

14 Binkert CA, Andrews RT, Kaufman JA. Utility of nonselective abdominal aortography in demonstrating ovarian artery collaterals in patients undergoing uterine artery embolization for fibroids. J Vasc Interv Radiol 2001;12(7):841-845 